\title{
Truth Detection in Social Media Posts using Jaccard Algorithm with SRTD and Word Net Concept
}

\author{
Priyanka Sangwan ${ }^{1}$, Rachna Behl ${ }^{2}$ \\ ${ }^{1}$ Research Scholar, MRIIRS Faridabad, Haryana, India \\ ${ }^{2}$ Asstant Professor, MRIIRS Faridabad, Haryana, India \\ 'er.priyankas1993@gmail.com , ${ }^{2}$ rachnabhel.fet@mriu.edu.in
}

\begin{abstract}
Counterfeit news has gotten an essential subject of exploration in an assortment of solicitations including semantics and programming building. In this work, clarification of how the issue is drawn nearer from the point of view of fundamental language managing, with the objective of building a framework to subsequently see misdirection in news. The rule challenge in this line of examination is gathering quality information, i.e., occasions of phony and true reports on a sensible dispersing of subjects. In this paper, a novel truth acknowledgment system with near words thoughts is added to the versatile and overwhelming truth disclosure structure used previously. By the use of practically identical words thoughts, the controlled fake news can be recognized with much basic and snappier. The features add up same meaning words which are compared using Jaccard algorithm in the main algorithm to detect a greater number of fake news with reliability score. The reliability score is calculated by combining independent score, attitude score and uncertainty score. The implemented software is found to be having better accuracy and results compared to existing truth detection methods.
\end{abstract}

Index Terms-Truth; big data, SRTD; Jaccard; Data Mining

\section{INTRODUCTION}

$\mathrm{O}$ NLINE media is critical these days. It is the best medium used for getting out the word which may be substantial or counterfeit. There is such a propensity for using web-based life arranges these days among people. [1] The substance introduced is normally related to ongoing advancements around people. [2] Sometimes misdirection may hurt the organization as it may be fundamental and may have horrendous results. In huge datasets it is hard to recognize talk or fake news. Thusly, it is imperative to execute web-based systems administration identifying structures and programming for disclosure of talk or double-dealing for real truth distinguishing proof in micro blogs containing huge data assessment. [3] This sets up the guideline focus of this paper to develop an item using sensible stage for disclosure of versatile truth-based news or any post using a fact score figuring. [4] Reality score in a general sense contains assessment of a score subject to free score, weakness score and air score examination of the post. [5] This is essentially the correct presently existing figuring which is named as adaptable and generous truth exposure computation used for examination of fake news in immense data distinguishing applications. [6] Current truth disclosure strategies don't thoroughly address the "confusion spread" issue where a noteworthy number of sources are spreading hoax data by methods for online frameworks organization media.[7] Many current truth exposure rely vigorously on the particular assessment of the devoted idea of sources, which reliably requires a sensibly enormous dataset. [8] Existing truth exposure plans don't thoroughly investigate the adaptability part of reality disclosure issue. [9] Therefore, it is needed to improve the at present existing truth disclosure computation for talk or fake new area in structure precision, capability, execution and execution of the proportionate in speedier speed. The essential objective of this paper is to perceive the assessment opening for truth disclosure estimations as explained here. Similarly, to improve the at present existing (Scalable and Robust Truth Discovery) SRTD count by changing the figuring to process reality score in such a way to improve reality distinguishing proof exactness and execution in gigantic data identifying applications. In Fig. 1, the customer case graph of the SRTD count for truth acknowledgment. In the enlightening assortment is moved, by then express scores are resolved to run the SRTD estimation with the help of the scores decided it will describe the posts given in dataset as clear or false with the execution time taken.

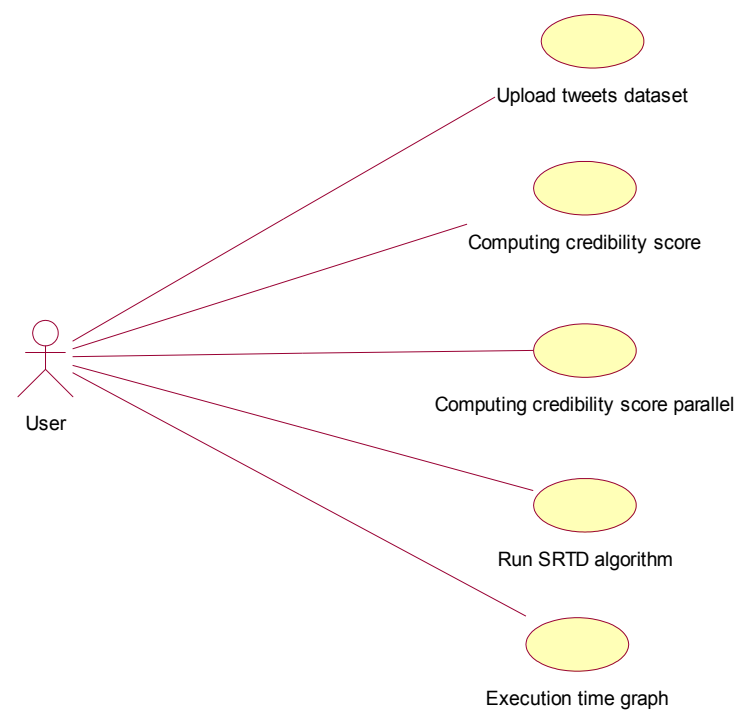

Fig.1. Use Case Diagram of SRTD Algorithm for Truth Detection 
There exist a few specific challenges to join the subject importance feature of cases into the truth disclosure courses of action. [10] To start with, Twitter is an open information duty stage where the source steadfastness (the likelihood of a source to report right cases) and the source point care (the likelihood of a source to report subject critical cases) are much of the times darken from the prior. [11] Second, it isn't immediate to recognize a predefined set of catchphrases (e.g., the hashtags on Twitter) to undeniably organize theme relevant cases from the point unnecessary ones considering the way that: the predefined watchwords may not so much appear in all point appropriate tweets (e.g., different words can be used to portray a comparative event on Twitter); subject unessential tweets can similarly contain the predefined catchphrases (e.g., to secure open thought). [12] Creator uses a truth disclosure-based convolution neural framework model for envisioning the legitimacy of Quora questions informational index. [13] The issue is that given a dataset of Quora questions one needs to recognize the toxic substance in the substance and gathering them into genuine request or not a bona fide request. Hurtful or misleading substance in the above issue is to perceive questions that have objective tone, stigmatizing information or isn't grounded really. [14] Recognizable evidence and portrayal of such substance is our essential models. Information agents in coherent, government, present day, and business zones face the trial of adjusting to rapidly creating volumes of information that are assembled in different applications. [15]

In this work, procedure to develop a Scalable and Robust Truth Discovery (SRTD) plan to address the lie spread, information sparsity, and adaptability challenges in huge information online life identifying applications. For truth detection, the basic algorithm generally uses only feature matching, which consists of words related to fake news and also, the generation of information is taken into consideration. Sometimes fake news is published using different words which have the same meaning, which was considered fake in the truth detection system. The existing system cannot detect this kind of information. The need to solve this problem is essential as the power of social media has become to produce any news; fake news may result in bad consequences as seen in the existing issues of the country. This problem is addressed in this thesis by adding word net concepts, which detects all kind of synonyms for feature matching algorithm, including the reliability score detection through sentiment, credibility and various other scores. Parallel execution is also implemented for the execution time improvement in the current work.

\section{IMPLEMENTATION}

The current algorithm cannot completely detect true news, because there is no knowledge of words or dictionaries, where the tweet includes a synonym of the same word. In order to overcome this, word net combinations are applied to the existing algorithm to increase its efficiency and address current algorithm problems with a reliability score with three scores independent, uncertainty and attitude score. By parallel execution of the ratings, the execution time can also be increased.

In this area, execution ideas of the application programming are clarified. A methodology is made to distinguish truth in social average tweets in huge information ideas, in view of a truth revelation calculation. The endeavor is to for the most part improve the execution time of handling of the information. A calculation is executed in particular adaptable and hearty truth disclosure SRTD to recognize the truth factor in the tweets. This relies upon a few score factors like unwavering quality, autonomous score, etc. Here there are three ideas from which truth can be find, for example, deception spread (people groups adding to bogus cases), information sparsity (deficient proof from huge dataset) and SRTD Algorithm.

Over two focuses can be determined utilizing 'Processing Credibility Score' and this score comprises of three sections 1) Attitude Score

\section{2) Uncertainty Score}

\section{3) Independent Score}

Attitude Score: utilizing opinion examination we can check whether tweets contain any negative estimations and if contains we will relegate less score, for example, - 1 and on the off-chance that no negative feelings, at that point will dole out 1 score.

Uncertainty Score: on the off-chance that two tweets are comparable, at that point tweets guarantee to be truthful and can relegate high score as 1 and not comparative methods 1 will appoint. Similitude will be check utilizing Jaccard separation and every single comparative tweet will be in same article.

Independent Score: on the off-chance that tweet just duplicate and retweet, at that point it will consider as reliant tweet which implies client isn't adding anything to its and will consider or guarantee as untruthful and will allot less score - 1 and every single autonomous tweet will have 1 score as its created by client by including some substance and guarantee it.

With all score a grid will frame up and this lattice will summarize to get unwavering quality score utilizing SRTD calculation, on the off-chance that dependability is high, at that point tweet is genuine in any case bogus. The work is effectively actualized utilizing java.

In this proposed function as EXTENSION, utilizing WORDNET to extricate comparable ideas from a word, comparable ideas mean discovering equivalent of given word, at some point in tweets people groups will utilize complex words whose significance might be don't know to certain clients and they discover hard to track down truth of tweets, this application additionally in propose work not utilizing any equivalent words coordinating to remove truth from tweets. 


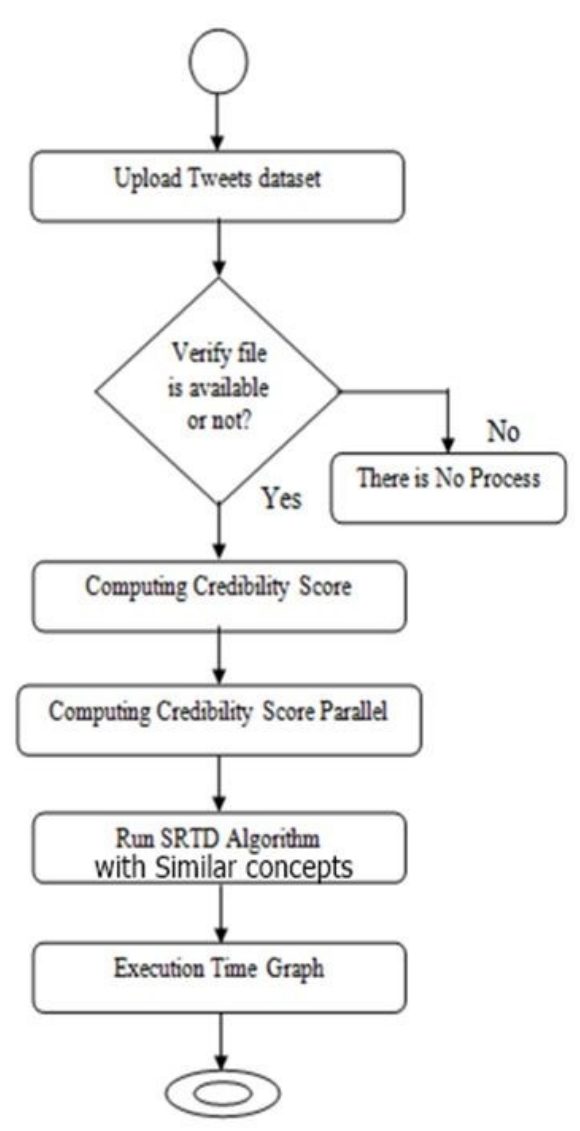

Fig. 2: Proposed Algorithm Activity Diagram

For instance, 'red' word is likewise called as 'dark red' and by utilizing ordinary compatibility or by applying notion application can't anticipate assessments appropriately and by giving interchange comparable words we can grow application expectation of truth in better manner.

'Pooch' comparative word is 'canine'

Like above model anybody can get comparable expressions of each word from tweet

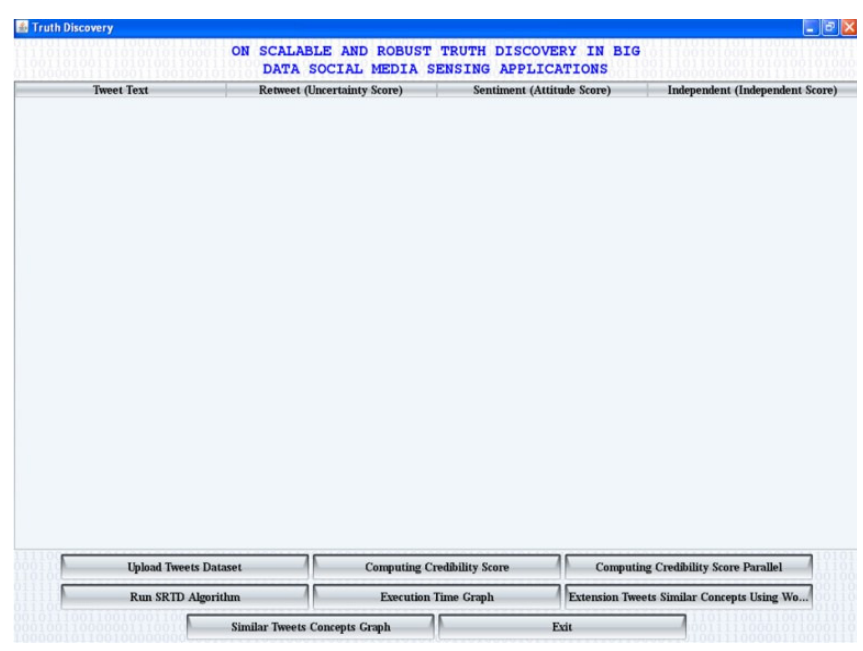

Fig.3: Software Screenshot main GUI Proposed
So to defeat from this issue in augmentation we included comparative idea extraction of given word utilizing WORDNET to get every single comparable word so client can comprehend accurate importance of complex words from given comparative words. WORDNET checks every comparative word and exactness of the current approach is expanded essentially. Fig 2: Shows the action chart of the progression of venture.

\section{RESULTS}

In this section, the results are mentioned for the implementation discussed in the previous section.

In above screen Fig.3, run all modules then click on 'Extension Tweets Similar Concepts Using Wordnet button to extract similar words from all tweets. See below screen shots.

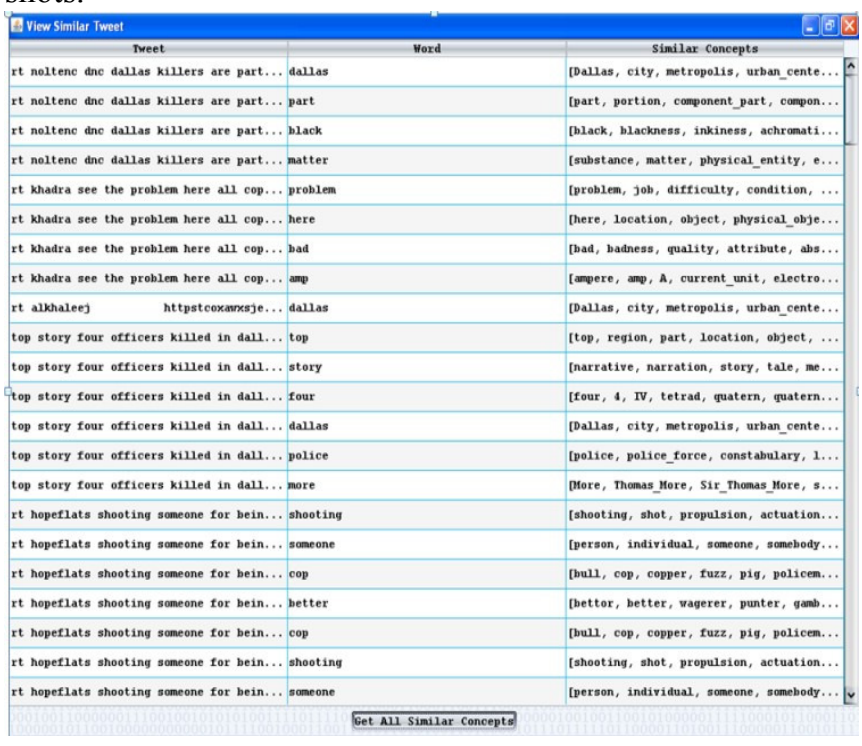

Fig.4: Similar Tweet Dataset

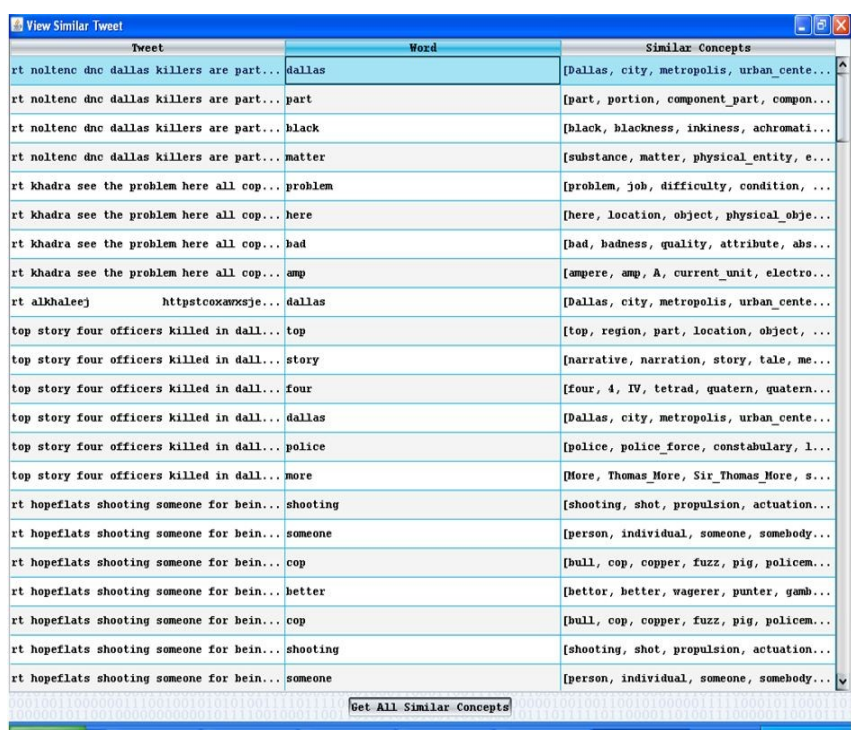

Fig.5: Selection of tweet 
In above screen Fig. 4 first column contains entire tweet and second column contains word from that tweet and third column contains all similar words of that second column word. In above screen we can for word Dallas the similar word can be city also. If one wants to see whole list of words then select any row from above screen table and click on 'Get All Similar Concepts' button to view complete list. See below screens

In above screen Fig.5, selection of first row through mouse now click on button to get below screen

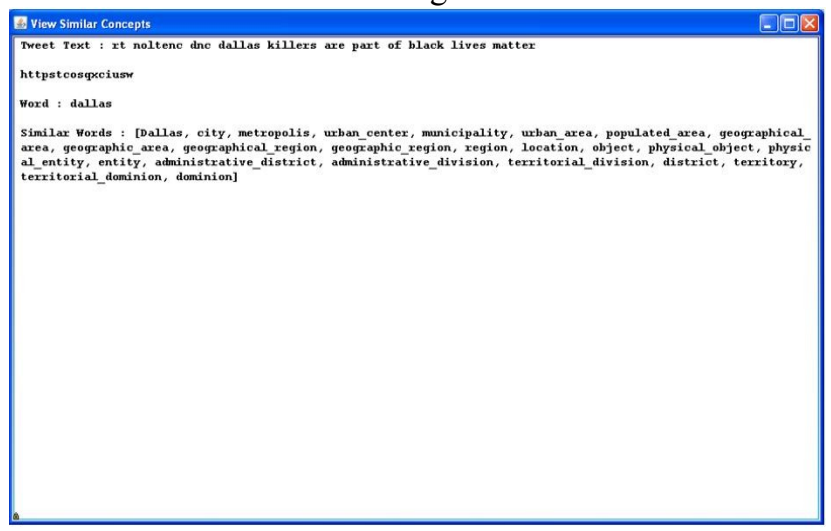

Fig.6: Similar Words Concept Screen

In above screen Fig. 6 we can see all similar words for given word Dallas.

Now click on 'Similar Tweets Concepts Graph' button to view graph which show number of similar word found for each tweet which detects the truth better.

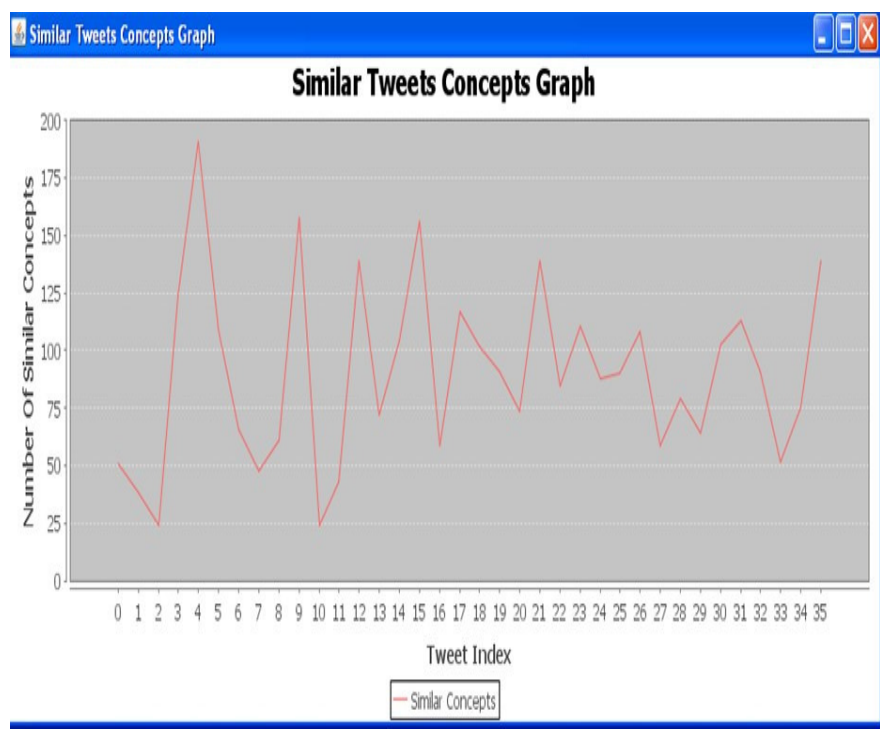

Fig.7: Similar Tweet Chart

In above graph Fig.7, x-axis represents tweet id and $y$-axis represents number of similar words for that tweet.

While adding the similar concepts to the existing truth detection scheme, the reliability score is improved and is better in case of the proposed work. The similar tweet detected also identifies the posts which were not earlier detected as the false posts as synonyms are used and thereby improving the accuracy of the existing system. This is also shown in figure 7 .

\section{CONCLUSION}

The role of truth detection in big data using Java was implemented in this thesis. A new strategy is being suggested for the tweeting using WORDNET dictionary to take into account related terms in a score improvement that helps to find truth better. This approach can also be used to enhance the exploration of reality by similar terms. A number of selected tweets display similar characteristics in relation to fake news using a similar definition. In the current process, the execution time is also increased by introducing a parallel running programmed instead of performing and scores normally.

\section{REFERENCES}

[1] Zhang, Daniel Yue \& Wang, Dong \& Vance, Nathan \& Zhang, Yang \& Mike, Steven. (2018). On Scalable and Robust Truth Discovery in Big Data Social Media Sensing Applications. IEEE Transactions on Big Data. PP. 1-1. 10.1109/TBDATA.2018.2824812.

[2] Zhang, Daniel Yue \& Han, Rungang \& Wang, Dong \& Huang, Chao. (2016). On robust truth discovery in sparse social media sensing. 1076-1081. 10.1109/BigData.2016.7840710.

[3] M. Nigade, M. Raut, P. Mane, S. Phadatare, "Truth Discovery in Big Data Social Media Application" Page 40-44 (C) Journal of Data Mining and Knowledge Engineering 2019

[4] Shihang Wang, Zongmin Li, Yuhong Wangand Qi Zhang, " Machine Learning Methods to Predict Social Media Disaster Rumor Refuters", Int. J. Environ. Res. Public Health 2019, 16, 1452; doi:10.3390/ijerph16081452

[5] Mohammed A-Sarem, Wadii Boulila, Muna Al-Harby, Junaid Qadir, and Abdullah Alsaeedi, "Deep Learning Based Rumor Detection on Microblogging Platforms: A Systematic Review", IEEE, 2019

[6] Cao, Juan \& Guo, Junbo \& Li, Xirong \& Jin, Zhiwei \& Guo, Han \& Li, Jintao. (2018). Automatic Rumor Detection on Microblogs: A Survey.

[7] Stefan Stieglitza,*, Milad Mirbabaiea, Björn Rossa, Christoph Neubergerb, "Social media analytics - Challenges in topic discovery, data collection, and data preparation", International Journal of Information Management, 2018

[8] Kai Shuy, Amy Slivaz, Suhang Wangy, Jiliang Tang, and Huan Liuy" Fake News Detection on Social Media: A Data Mining Perspective", SIGKDD Explorations Volume 19, Issue 1

[9] Carlos Argueta, Yi-Shin Chen, "Multi-Lingual Sentiment Analysis of Social Data Based on Emotion-Bearing Patterns", Proceedings of the Second Workshop on Natural Language Processing for Social Media (Social NLP), pages 38-43,Dublin, Ireland, August 242014

[10] Trisha Dowerah Baruah, "Effectiveness of Social Media as a tool of communication and its potential for technology enabled connections: A micro-level study", International Journal of Scientific and Research Publications, Volume 2, Issue 5, May 20121 ISSN 2250-3153

[11] N. Baggyalakshmi, Dr. A. Kavitha, Dr. A. Marimuthu, "Microblogging in Social Networks - A Survey", International Journal of Advanced Research in Computer and Communication Engineering, ISO 3297:2007

[12] Certified Vol. 6, Issue 7, July 2017 
[13] Jiawei Zhang1, Bowen Dong2, Philip S. Yu, "FAKEDETECTOR: Effective Fake News Detection with Deep Diffusive Neural Network", arxiv, 2018

[14] Shuo Yang,yz Kai Shu,z SuhangWang,x Renjie Gu,y Fan Wu,y Huan Liuz "Unsupervised Fake News Detectionon Social Media: A Generative Approach", The Thirty-Third AAAI Conference on Artificial Intelligence (AAAI-19)
[15] Conroy, Nadia \& Rubin, Victoria \& Chen, Yimin. (2015). Automatic deception detection: Methods for finding fake news. Proceedings of the Association for Information Science and Technology. 52. 110.1002/pra2.2015.145052010082.

[16] Zhou, Xinyi \& Zafarani, Reza. (2018). Fake News: A Survey of Research, Detection Methods, and Opportunities. 